\title{
Immunonutrition Recommendations for Coronavirus Infection
}

\author{
Dr. Harmanjot Kaur ${ }^{1 *}$, Dr. Roopjot ${ }^{2}$ \\ ${ }^{I}$ Diet \& Nutrition Consultant, SGS Diets, Khanna, Punjab, India \\ ${ }^{2}$ Director, Physician \& Nutritionist, SGS Diets, Khanna, Punjab, India
}

*Corresponding Author: Dr. Harmanjot Kaur, Diet \& Nutrition Consultant, SGS Diets, Khanna, Punjab, India.

\begin{abstract}
An epidemic of a novel corona virus (COVID-19 or 2019-CoV) infection has posed remarkable risk to international health and economy. As there is no definite therapy for this COVID-19 virus, there is a vital need to find alternative methods to control the spread of disease. Nutritional intervention can help prevent the occurrence of active infection. Foods abundant in zinc, omega-3 fatty acids, vitamin $C$ and probiotics help in prevention and treatment of human corona virus infection. Warming foods also play an important role in boosting human immunity against viral infections. A balanced diet comprising nutrient-rich vegetables, fruits, pulses, cereals and curd along with healthy lifestyle is key point to boost the immune system in the fight against COVID-19.
\end{abstract}

Keywords: Anti-inflammatory, anti-oxidative, ginger, Nutrition, COVID-19

\section{INTRODUCTION}

Nutrition is a key determinant of health and plays important role in the treatment regimen for acute and chronic diseases and applies particularly to infections for which treatment has not yet been discovered and validated. The 2014-2016, Ebola virus outbreak in Western Africa demonstrated that immediate supportive care significantly reduces case fatality rates. This may help in current SARS-CoV-2 (or COVID-19) pandemic that is devastating the world. Emerging evidence shows that COVID19 is associated with negative outcomes in older, co-morbid and hypoalbuminemic patients $[1,2]$. When considered jointly, the emerging literature on patients with COVID-19 indirectly highlights the relevance of nutrition in possibly determining their prognosis. Older age and the presence of co-morbid conditions are almost invariably associated with impaired nutritional status and sarcopenia, independently of body mass index. Interestingly, a high body mass index score appears to be related to a poor prognosis in co morbid patients with COVID19, which further adds to a possible role of sarcopenic obesity in influencing outcome [3]. Also, lymphopenia, which is a marker of malnutrition, is a negative prognostic factor in patients with COVID-19. Albumin circulating levels should not be considered as a nutritional marker in patients with inflammatory response, but a recent report that a low pre albumin level predicts progression to acute respiratory distress syndrome (ARDS) suggests that poor nutritional intake contributes to the outcome. Nutritional intervention appears to be critical because most patients rapidly progress from cough to dyspnea and to respiratory failure and then need mechanical ventilation support for survival $[4$, 5]. Nutritional status appears to be most critical factor influencing the outcome of patients with COVID-19, but not much information has emerged so far on the impact of early nutritional support in pre ICU patients with COVID-19. The National Health Commission of the People's Republic of China and the National Administration of Traditional Chinese Medicine recommend implementing strengthened supportive care to ensure sufficient energy intake. It was recently elaborated in another study on a list of nutrients with possible anti-corona virus effects based on in vitro and clinical studies [6]. A protocol based on the clinical experience learned from the daily challenges posed by patients with COVID-19 may help stimulate discussions regarding nutrition-delivery options. 


\section{NUTRITIONAL RECOMMENDATIONS DURING COVIID-19 PANDEMIC}

According to the patients' condition, provide high protein, high vitamin and carbohydrate containing diets (e.g. eggs, fish, lean meat, milk, etc.) for enough nutrition to improve physical condition. Those patients who cannot eat but are compatible with enteral nutrition should be given enteral nutrition as soon as possible. For the patients incompatible with enteral nutrition, parenteral nutrition should be given timely to meet energy requirements [7].

\subsection{Individual}

- Try to eat well-balanced diets, avoid irregular snacking.

- Choose foods rich in vitamins A, C, E, B6 and $\mathrm{B} 12$, zinc and iron including citrus fruits, dark green leafy vegetables, nuts, and dairy products.

- Maintain a healthy lifestyle of exercise (homeexercises), regular sleep and meditation.

- Avoid smoking, alcohol, and drugs.

- Refrain from spreading misinformation related to nutrition and dietary intake and COVID-19.

\subsection{Community}

- Spread awareness regarding the devastating consequences of hoarding and panic buying.

- Identify and support populations at risk of malnutrition within the community especially elderly and patients with chronic diseases.

- Create a structured and reliable support system to ensure availability, access and affordability of essential food commodities to all members of the community.

\subsection{National}

- Define, finance and distribute a food basket of least-cost diet that provides the health needs of the population, ensures the use of local agricultural produce of the country and minimizes reliance on food imports.

- Mobilize resources in order to finance food purchases and provisions.

- Waive taxation for staple foods and commodities.

- Support agricultural and food production industries.

- Closely monitor and inspect food prices and markets.
- Build networks with the private sector, the international agencies and local communities.

- Maintain high levels of transparency, critical to build trust, support and compliance.

\subsection{Global}

- Assure continuous flow of global trade, avoiding any trade restrictions would be beneficial to keep food and feed supplies as well as those of agricultural inputs from worsening local conditions already strained by COVID-19 response measures.

- Reduce import tariffs and other restrictions on food commodities. [8]

\section{NUTRITIONAL INTERVENTIONS}

\subsection{Ginger}

Ginger has anti-inflammatory properties which play an important role to combat a cold or flu. Inflammatory disorders such as gastritis, esophagitis and hepatitis which are caused not only by infectious agents such as viruses, bacteria and parasites also by physical and chemical agents like heat, acid, cigarette smoke and foreign bodies are recognized as risk factors for human cancer. Because inflammation can affect body's immune system, anti-inflammatory ginger can play a key role in boosting immunity [9].

\subsection{Selenium}

Nutritional status plays a very important role in the protection against infectious diseases. Deficiency leads to not only the impaired immune response but also the viral pathogen itself. Dietary selenium deficiency that causes oxidative stress in the host can alter a viral genome so that a normally benign or mildly pathogenic virus can become highly virulent in the deficient host under oxidative stress. It had reported that selenium deficiency could not only increase the pathology of an influenza virus infection. It is because that selenium could assist a group of enzymes along with vitamin $\mathrm{E}$ to prevent the formation of free radicals and prevent oxidative damage of cells and tissues. It was reported that synergistic effect of selenium with ginseng stem leaf saponins could induce immune response to a live bivalent infectious bronchitis corona virus vaccine in chickens. Therefore, selenium supplementation could be an effective choice for the treatment of this novel virus of COVID-19 $[6,10]$. 


\subsection{Zinc}

Zinc is a dietary trace mineral important for the maintenance and development of immune cells of both the innate and adaptive immune system. Zinc deficiency results in dysfunction of both humoral and cell-mediated immunity and increases susceptibility to infectious diseases. Zinc supplement given to zinc deficient children could reduce measles related morbidity and mortality caused by lower respiratory tract infections. In addition, the combination of zinc and pyrithione at low concentrations inhibits the replication of SARS corona virus (SARS-CoV). Therefore zinc supplement may have effect on COVID-19 and related symptoms like diarrhea and lower respiratory tract infection $[11,12]$.

\subsection{Vitamin C}

Vitamin C was first purified in the early 1930s and soon after physicians proposed that vitamin $\mathrm{C}$ could be beneficial in the treatment of pneumonia. Historically, vitamin $\mathrm{C}$ has been used for immune support and is known to be acquired by neutrophils. This nutrient supports epithelial barrier function against pathogens, cellular functions of the adaptive and innate immune systems, and protects against oxidative stress [13, 14]. High-dose vitamin C (3-5 g/d) shortens the use time of booster drugs and ventilator is effective for acute respiratory distress syndrome that significantly reduces mortality [15]. Another study shows that by administration of one of the highest dosages, 2 $\mathrm{g} /$ day, to US Marine recruits for 2 months with no reported symptoms attributed to the pills. In this study there was a reduced incidence of pneumonia among the recruits receiving vitamin $\mathrm{C}$, but only 8 of 674 had pneumonia, indicating a need for larger sample sizes [16].

\subsection{Vitamin D}

A number of studies have investigated interrelationships between vitamin D and its effects on respiratory viruses and conditions such as community-acquired pneumonia. It is well appreciated that vitamin $\mathrm{D}$ is a powerful immune regulator, with vitamin $\mathrm{D}$ receptors being expressed by the majority of immune cells (B and $\mathrm{T}$ lymphocytes, macrophages and monocytes). It has also been proposed that immune cells themselves can convert $25(\mathrm{OH})$ D3 into 1, 25(OH) 2D3, its active form [17]. A systematic review and meta- analysis 39 has collated evidence from 25 separate randomized controlled trials $(\mathrm{n}=11321$ participants $)$ studying the effects of vitamin D supplementation on acute respiratory infections among those aged 0-95 years. Protective effects were seen among all participants, but particularly among those with baseline 25hydroxyl vitamin D levels $<25 \mathrm{nmol} / \mathrm{L}$, indicative of deficiency. Overall, the authors concluded that vitamin D appeared to be a safe strategy to protect against acute respiratory tract infections [18].

The administration of other micronutrients in doses higher than the recommendations should only be carried out if a specific deficiency is present. For example, thiamine, functional to energy metabolism, must be integrated from the 8th-10th day of hospitalization. It can be administered thiamine $100 \mathrm{mg}$ intramuscular every 24 hours for 5 days. Immuno nutrients have shown to have a considerable influence on immune function and improve metabolic and nutritional indices such as nitrogen balance and serum proteins. There are several types of immune nutrients such as arginine, nucleotides, glutamine, $\omega-3$ fatty acids, and their functions and mechanisms are different. Immuno nutrients can promote patient recovery by inhibiting inflammatory responses and regulating immune function $[19,20]$.

At this time of the COVID-19 pandemic, given the huge sanitary cost and human resource spending, attention to nutritional medical therapy and nutritional status should be considered among the "basic vital signs" as important as blood pressure and pulse oximetry. The suggestions for a correct evidence-based nutritional therapy here reported are indicated for critically COVID-19 patients and should be included among lifesaving therapies.

\section{CONClusion}

In conclusion, the influence of this COVID-19 pandemic on nutrition and dietary intake has already gone beyond the individual and community to reach national and global levels. A particular feature of this pandemic is highlighting the interdependence of these various levels, whereby the health of the individual became a direct function of own awareness and choices, the unity of the community, the preparedness of the government and ultimately the global engagement. In this perspective, a framework for action and recommendations is presented at each of that level. The main goal of these recommendations 
is to maintain the physical and mental health of individuals, resilience of communities and national and global food security. Micronutrients (vitamins and minerals) and phytonutrients that are primarily available in fruits, nuts, vegetables and whole grains enhance both native and adaptive immune function and prevent infection among others.

\section{REFERENCES}

[1] Covinsky K, Katz M. Supplemental nutrition assistance program - Do not take the food out of patients' mouth. JAMA Intern Med 2020.

[2] Zhou F, Yu T, Du R, Fan G, Liu Y, Liu Z, et al. Clinical course and risk factors for mortality of adult in patients with COVID-19 in Wuhan, China: A retrospective cohort study. Lancet 2020; 395:1054-62.

[3] Peng YD, Meng K, Guan HQ, Leng L, Zhu RR, Wang BY, etal .Clinical characteristics and outcomes of 112 cardiovascular disease patients infected by 2019-nCoV. Zhonghua Xin Xue Guan Bing Za Zhi 2020; 48:E004

[4] Arentz M, Yim E, Klaff L, Lokhandwala S, Riedo FX, Chong M, et al. Characteristics and outcomes of 21 critically ill patients with COVID-19 in Washington State. JAMA 2020.

[5] Wu C, Chen X, Cai Y, Xia J, Zhou X, Xu S, et al. Risk factors associated with acute respiratory distress syndrome and death in patients with Corona virus Disease 2019 pneumonia in Wuhan, China. JAMA Intern Med 2020.

[6] Zhang L, Liu Y. Potential interventions for novel corona virus in China: A systematic review. J Med Virol 2020; 92:479-90.

[7] Harmanjot Kaur and Roopjot (2020). Human Corona Virus Infection and Role of Nutrition. CPQ Nutrition, 4(1): 01-07.

[8] Naja, F., Hamadeh, R. Nutrition amid the COVID-19 pandemic: a multi-level framework for action. Eur J Clin Nutr (2020).

[9] Mashhadi NS, Ghiasvand R, Askari G, Hariri M, Darvishi L, Mofid MR. Anti-oxidative and anti-inflammatory effects of ginger in health and physical activity: review of current evidence. Int J Prev Med. 2013; 4(Suppl 1):S36-S42.

[10] Ma X, Bi S, Wang Y, Chi X, Hu S. Combined adjuvant effect of ginseng stem-leaf saponins and selenium on immune responses to a live bivalent vaccine of Newcastle disease virus and infectious bronchitis virus in chickens. Poult Sci. 2019; 98:3548-3556.

[11] Maares M, Haase H. Zinc and immunity: an essential interrelation. Arch Biochem Biophys. 2016; 611:58-65.

[12] Awotiwon AA, Oduwole O, Sinha A, Okwundu CI. Zinc supplementation for the treatment of measles in children. Cochrane Database Syst Rev. 2017;2017(6):CD011177

[13] Liugan M, Carr AC, Vitamin C. Vitamin C and neutrophil function: findings from randomized controlled trials. Nutrients 2019; 11:2102. 28

[14] Carr A, Maggini S. Vitamin C and immune function. Nutrients 2017; 9:1211.

[15] Derbyshire E, Delange COVID-19: is there a role for immunonutrition, particularly in the over 65s? J. BMJNPH 2020; 0:1-6.

[16] Zdrenghea MT, Makrinioti H, Bagacean C, et al. Vitamin D modulation of innate immune responses to respiratory viral infections. Rev Med Virol 2017; 27:e1909.

[17] Telcian AG, Zdrenghea MT, Edwards MR, et al. Vitamin D increases the antiviral activity of bronchial epithelial cells in vitro. Antiviral Res 2017; 137:93-101.

[18] Martineau AR, Jolliffe DA, Hooper RL, et al. Vitamin D supplementation to prevent acute respiratory tract infections: systematic review and meta- analysis of individual participant data. BMJ 2017; 356:i6583.

[19] Marik Pe, Khangoora V, Rivera R, Hooper MH, Catravas J. Hydrocortisone, vitamin C, and thiamine for the treatment of severe sepsis and septic shock: a retrospective before-after study. Chest 2017; 151: 1229-1238.

[20] Rosenthal Md, Carrott Pw, Patel J, Kiraly L, Martindale Rg. Parenteral or enteral arginine supplementation safety and efficacy. J Nutr 2016; 146: 2594S-2600S.

Citation: Dr. Harmanjot Kaur, Dr. Roopjot. Immunonutrition Recommendations for Coronavirus Infection. ARC Journal of Nutrition and Growth. 2020; 6(1):23-26. DOI: dx.doi.org/ 10.20431/2455-2550.0601004.

Copyright: (C) 2020 Authors. This is an open-access article distributed under the terms of the Creative Commons Attribution License, which permits unrestricted use, distribution, and reproduction in any medium, provided the original author and source are credited. 